Revue internationale P.M.E.

Économie et gestion de la petite et moyenne entreprise

Revue

internationale

PME

\title{
Stratégies d'adoption d'une démarche qualité en PME
}

\section{Karim Messeghem et Nicolas Varraut}

Volume 11, numéro 1, 1998

URI : https://id.erudit.org/iderudit/1009038ar

DOI : https://doi.org/10.7202/1009038ar

Aller au sommaire du numéro

Éditeur(s)

Presses de l’Université du Québec

ISSN

0776-5436 (imprimé)

1918-9699 (numérique)

Découvrir la revue

Citer cette note

Messeghem, K. \& Varraut, N. (1998). Stratégies d'adoption d'une démarche qualité en PME. Revue internationale P.M.E., 11(1), 101-122.

https://doi.org/10.7202/1009038ar

\section{Résumé de l'article}

Dans le cadre de cette contribution, nous proposons une analyse du processus de décision d'adoption d'une démarche qualité par le dirigeant de PME. L'approche cognitive que nous retenons met l'accent sur l'articulation entre vision stratégique et intention stratégique. Elle nous permet de faire ressortir la diversité des stratégies d'adoption d'une démarche qualité en PME. L'accent est mis sur deux stratégies dominantes : la stratégie proactive, dont le principal objectif est la recherche d'un avantage concurrentiel, et la stratégie réactive, qui apparaît comme une réponse à de nouvelles exigences ou au comportement de concurrents amorçant eux-mêmes une démarche qualité Nous privilégions dans cet article l'assurance qualité. En effet, cette démarche qualité semble de plus en plus s'imposer dans les relations entre grandes entreprises et PME. 


\title{
Stratégies d'adoption d'une démarche qualité en PME
}

\author{
Karim MESSEGHEM \\ Nicolas VARRAUT \\ Université de Montpellier I
}

\author{
MOTS CLÉS
}

\section{Processus de décision - Vision stratégique - Intention stratégique Assurance qualité - Dirigeant de PME}

\begin{abstract}
RÉSUMÉ
Dans le cadre de cette contribution, nous proposons une analyse du processus de décision d'adoption d'une démarche qualité par le dirigeant de PME. L'approche cognitive que nous retenons met l'accent sur l'articulation entre vision stratégique et intention stratégique. Elle nous permet de faire ressortir la diversité des stratégies d'adoption d'une démarche qualité en PME. L'accent est mis sur deux stratégies dominantes: la stratégie proactive, dont le principal objectif est la recherche d'un avantage concurrentiel, et la stratégie réactive, qui apparaît comme une réponse à de nouvelles exigences ou au comportement de concurrents amorçant eux-mêmes une démarche qualité. Nous privilégions dans cet article l'assurance qualité. En effet, cette démarche qualité semble de plus en plus s'imposer dans les relations entre grandes entreprises et PME.
\end{abstract}

\section{LES AUTEURS}

Karim Messeghem, attaché temporaire d'enseignement et de recherche (ATER) à l'Université de Montpellier III, enseigne le contrôle de gestion et la gestion financière et intervient en gestion de la qualité. Il est membre de l'ERFI (Équipe de recherche sur la firme et l'industrie) où il mène une recherche doctorale sur l'assurance qualité en PME. Adresse: Karim MESEGHEM, Amphitéâtre II - App. 608, 55, Place Vigne d'Octon, 34000 Montpellier, Tél. : 0467993117.

Nicolas Varraut, attaché temporaire d'enseignement et de recherche (ATER) à l'IUT de Mulhouse, enseigne la comptabilité générale et l'analyse financière. Il est membre de l'ERFI (Équipe de recherche sur la firme et l'industrie) au sein de laquelle il conduit une recherche doctorale sur la vision stratégique du dirigeant de PME. 


\begin{abstract}
In this paper, we present an analysis of the decision-making process by the manager of small and medium-sized firms in order to adopt a quality approach. The cognitive approach we consider emphasizes the link between strategic vision and strategic intent. Such approach enables us to highlight the diversity of the possible strategies to make a quality approach in small and medium-sized firms. We emphasize two main strategies : a proactive strategy whose main purpose is to find a competitive advantage and a reactive strategy which appears to meet new requirements or correspond to the behavior of competitors who are also adopting a quality approach. In this paper, we focus rather on quality insurance as it seems to be now essential in the relations between large firms and small and medium-sized firms.
\end{abstract}

\title{
RESUMEN
}

En este artículo, proponemos analizar el proceso de decisión de los dirigentes de PyMEs para adoptar un gestión de la calidad. El estudio cognitivo que tomamos en cuenta pone de relieve la relación entre visión estratégica e intención estratégica. Nos permite subrayar la diversidad de las estrategias que permitan adoptar una gestión de la calidad en las PyMEs. Se subrayan dos estrategias principales: la estrategia proactiva cuyo objetivo principal es encontrar una ventaja en la competencia y la estrategia reactiva que parece corresponder a nuevas necesidades o al comportamiento de los competidores que también están adoptando una gestión de la calidad. En este artículo, se privilegia el aseguramiento de la calidad porque parece que cada vez más se impone dentro de las relaciones entre las grandes empresas y las PyMEs.

\section{Introduction}

Les démarches qualité en PME font l'objet d'une attention accrue dans la littérature (Esposito et Raffa, 1996; Ghobadian et Gallear, 1996; Gunasekaran et al., 1996; Guillon et Weill, 1997). La littérature sur la gestion de la qualité a longtemps été centrée sur la grande entreprise. Cependant, les réflexions s'orientent de plus en plus vers la petite entreprise. On peut expliquer en partie ce gain d'intérêt par l'enjeu stratégique que représente très souvent la qualité dans les relations entre petites et grandes entreprises (Baudry, 1995 ; Messeghem, 1997). L'assurance qualité apparaît comme la principale approche retenue par les PME qui souhaitent se lancer dans une démarche qualité, comme le révèle l'enquête conduite par Guillon et Weill (1997) auprès de PME. Cette forme de gestion de la qualité s'inscrit le plus souvent dans les relations interentreprises comme un moyen d'accroître la confiance. L'adoption d'un système d'assurance de la qualité peut également remplir un objectif interne destiné à promouvoir un projet d'entreprise fédérateur et mobilisateur.

Cette contribution vise à mieux faire comprendre le processus de décision d'adoption d'une démarche qualité à partir d'une approche cognitive. Nous allons 
nous focaliser sur le dirigeant-propriétaire de petite entreprise. Certains travaux sur la PME montrent la difficulté de proposer une définition de cette notion au contour flou (Marchesnay, 1991 ; Julien, 1994, Torres, 1997). Nous retiendrons la définition de Julien (1994) sous forme de continuum. Cet auteur propose de retenir six caractéristiques pour mieux cerner le concept de PME : la petite taille, la centralisation de la gestion, une faible spécialisation, une stratégie intuitive ou peu formalisée, un système d'information interne peu complexe ou peu organisé, un système d'information externe peu complexe. Dans les PME, le dirigeant a tendance à être au centre du système de gestion de l'entreprise et à l'influencer à travers sa vision (Marchesnay, 1991). La notion de vision stratégique sera au cœur de notre réflexion. On peut considérer la vision stratégique comme une représentation du devenir de l'entreprise pour le dirigeant. Cette notion est particulièrement importante pour comprendre la stratégie de l'entreprise. On peut d'ores et déjà noter que le problème de la qualité peut s'intégrer dans une stratégie plus globale. La qualité semble donc correspondre seulement à un aspect de la vision stratégique, un aspect qui peut jouer un rôle majeur ou mineur selon le dirigeant et selon le contexte. La décision de faire certifier son entreprise peut s'inscrire dans une stratégie proactive de recherche d'avantage concurrentiel. Mais elle peut également s'inscrire dans une stratégie réactive qui répondrait aux exigences des donneurs d'ordres, des clients, de la réglementation ou qui ferait suite à la certification d'un concurrent.

Dans le cadre de cette communication, nous allons essayer de proposer un cadre conceptuel destiné à mieux éclairer le processus de décision de mise sous assurance qualité. Dans une première partie, nous présenterons le cadre conceptuel retenu qui s'appuie en partie sur le modèle consacré à l'entrepreneuriat proposé par Shapero (1982). En deuxième partie, nous insisterons sur l'articulation entre les concepts de vision stratégique et d'intention stratégique. Enfin, dans la dernière partie, nous nous attacherons à montrer la pluralité des démarches et donc des parcours cognitifs possibles suivant les dirigeants.

\section{Proposition d'un cadre conceptuel}

L'objet de cette première partie vise à clarifier les notions de vision stratégique et d'intention stratégique, puis de montrer comment elles peuvent s'articuler. Nous nous appuierons sur le modèle de Shapero (1982) pour analyser la décision d'adoption d'un modèle d'assurance de la qualité.

\subsection{Vision et intention stratégiques : deux notions distinctes}

Les notions de vision et d'intention stratégiques, en tant que concepts, ont fait l'objet de contributions à partir de la fin des années 1980. Bien qu' une différence de nature soit pressentie entre les notions de vision stratégique et d'intention stratégique, les auteurs, dans leur immense majorité, les utilisent indifféremment, sans les différencier 
sur le plan de leur nature. Nous pensons que ces deux notions sont susceptibles d'entretenir des relations dans l'esprit du dirigeant et qu'elles peuvent donc recouvrir des réalités proches.

Hamel et Prahalad (1989) sont les promoteurs de la notion d'intention stratégique (strategic intent). Une abondante littérature a repris les préceptes énoncés par ces auteurs pour enrichir et renouveler l'approche «resource-based». Notons que ces travaux sont pour la plupart centrés sur la grande entreprise et qu'ils n'adoptent pas une perspective cognitive. Il existe trois points essentiels sur lesquels se rejoignent les auteurs : l'intention est caractérisée par un état mental, elle combine buts et moyens, et elle comporte une dimension temporelle.

Traitant du comportement humain volontaire, Searle (1984) indique que « les actions se composent toujours de deux éléments, une composante mentale et une composante physique ». Il ajoute que « la composante mentale est une intention». Il précise que «si tout va bien, la composante mentale cause la composante physique et la représente». Nguyen-Xuan, Richard et Hoc (1990) indiquent que «le cours de l'activité est défini par une tâche en cours et par des tâches en instance ». Reprenant les travaux de Dörner (1986), ils définissent les intentions comme des tâches en instance.

Nous appuyant également sur les travaux de Bird (1988), nous choisissons de définir l'intention stratégique de la manière suivante :

\section{L'intention stratégique est un état mental qui dirige l'attention du dirigeant vers la recherche et la mise en place de moyens particuliers dans le but de réaliser un objet stratégique spécifique.}

Le terme vision a toujours été présent dans la littérature. Ainsi, lorsque Mintzberg (1973) décrit les trois modes de formation de la stratégie, il indique que dans le mode qualifié par lui d'entrepreneurial, « la stratégie est guidée par la vision personnelle du dirigeant ». C'est par l'intermédiaire de l'étude du leadership dans les grandes entreprises américaines (Zaleznik, 1977 ; Bennis, 1982) que la notion de vision s'est peu à peu hissée au rang de concept étudié pour lui-même. À partir de la fin des années 1980, cette notion a commencé à faire l'objet de contributions propres, notamment par l'intermédiaire de Filion (1989), D'Amboise et Gasse (1989), Westley et Mintzberg (1989) et Carrière (1990).

Tout au début des années 1990, le concept de vision est utilisé par la plupart des auteurs sans que de véritables réflexions soient menées sur la nature même de cette notion. Certains utilisent les notions de vision et d'intention stratégiques sans les différencier, implicitement (Parikh et Neubauer, 1993 ; Coulson-Thomas, 1992 ; D’Amboise et Bouchard, 1990 ; Gratton, 1994 ; Greffeuille, 1994a, 1994b, 1994c ; 
Nkongolo-Bakenda, D’Amboise et Garnier, 1993; Schoemaker, 1992; Smith, 1994 ; Tregoe, Zimmerman, Smith et Tobia, 1991) ou explicitement (Ramanantsoa, 1993). C'est sans doute l'une des raisons pour lesquelles le concept de vision stratégique est parfois critiqué. Harel Giasson (1995) qualifie même la notion de vision de «fourre-tout» conceptuel.

Il faut attendre des travaux comme ceux de Cossette (1994) qui retient une approche cognitive et qui utilise la cartographie cognitive afin d'étudier la vision stratégique de dirigeants de PME pour, même si cela est relativement implicite, poser la question de la nature véritable de cette notion. Cossette définit la vision stratégique comme un «produit cognitif constitué d'un réseau de concepts jugés importants pour l'avenir de l'entreprise. Elle met en évidence un système d' " explications » (causes ou moyens) et de «conséquences» (effets ou fins) qui guide les individus concernés dans l'interprétation des événements et dans les actions à entreprendre ». Cette définition s'appuie sur les travaux de Cossette et Audet (1994) qui indiquent qu'une «carte cognitive est une représentation graphique de la représentation mentale que le chercheur se fait d'un ensemble de représentations discursives énoncées par un sujet à partir de ses propres représentations cognitives à propos d'un objet particulier ».

Après avoir approfondi la perspective cognitive et procédé à l'observation dans la littérature des points communs des différentes définitions de la vision stratégique proposées par les auteurs, nous avons choisi de définir cette dernière de la façon suivante :

La vision stratégique du dirigeant est sa représentation mentale, à la fois du présent et du futur, de son organisation et de son environnement.

Une caractéristique importante à retenir est la dimension temporelle attachée à la vision stratégique. Nous verrons comment ce point peut permettre de relier la notion de vision stratégique à celle d'intention stratégique.

\subsection{Les apports du modèle entrepreneurial de Shapero}

Nous allons présenter un schéma qui met en relation la vision stratégique, l'intention stratégique et le comportement. Nous pensons que ces notions peuvent ne pas expliquer à elles seules le processus de prise de décision de faire certifier l'entreprise. C'est la raison pour laquelle nous avons emprunté un certain nombre d'éléments au modèle de Shapero (1982), lequel accorde une place centrale au concept d'intention pour expliquer la décision et le comportement entrepreneurial. Il ne qualifie pas l'intention de stratégique et son modèle n'intègre pas le concept de vision stratégique. Nous pensons cependant que les quatre éléments qu'il présente 
dans son modèle - désirabilité perçue, faisabilité perçue, propension à agir et événement précipitatif - sont susceptibles de compléter et d'enrichir notre grille d'analyse de l'articulation entre vision stratégique, intention stratégique et comportement. Shapero présente la désirabilité et la faisabilité perçues comme des variables médiatrices entre des influences exogènes et l'intention. Selon cet auteur, la propension à agir a une incidence exogène sur l'intention. L'événement précipitatif, quant à lui, est présenté comme une variable modératrice entre l'intention et le comportement. Précisons que notre schéma ne respecte pas tout à fait la structure et le contenu du modèle de Shapero. En effet, nous avons choisi d'explorer les relations que peuvent entretenir la vision et l'intention stratégiques dans l'esprit du dirigeant. Nous utilisons simplement une partie de la structure et donc un certain nombre d'éléments du modèle de Shapero pour étoffer notre réflexion.

La figure 1 illustre notre conception du processus d'articulation entre vision stratégique, intention stratégique et comportement de mise en œuvre, au sein duquel peut s'inscrire le processus relatif à la décision de faire certifier l'entreprise.

FIGURE 1

Processus d'articulation entre vision stratégique, intention stratégique et comportement

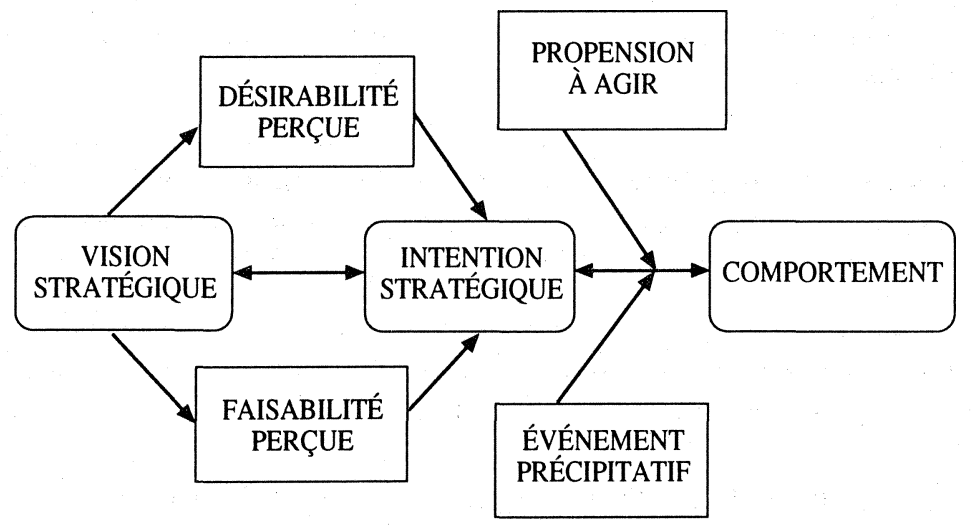

L'analyse de la relation (voire de l'interaction) qui peut exister entre vision et intention stratégiques peut être enrichie à l'aide des notions de désirabilité perçue, de faisabilité perçue et de propension à agir.

La faisabilité perçue par le dirigeant correspond, selon nous, à sa perception de sa capacité de mener à terme et avec succès, un projet qui s'intègre dans la composante « futur» de sa vision stratégique (sa représentation présente du futur). 
La désirabilité perçue s'apparente à la perception de son désir de voir aboutir son «projet». La propension à agir caractérise un trait de personnalité relatif au dynamisme du dirigeant et donc à sa capacité à mettre en œuvre les moyens nécessaires pour parvenir à ses fins. Nous considérons que c'est de la combinaison des influences de la vision stratégique (la tension qu'elle peut provoquer), de la faisabilité perçue, de la désirabilité perçue et de la propension à agir que pourra émerger une intention stratégique. Le passage de l'intention stratégique au comportement peut être expliqué par la force de l'intention (Bird, 1988; Dörner, 1986). NguyenXuan, Richard et Hoc (1990) indiquent que « la force d'une intention [...] dépend [...] de la proximité temporelle de la date limite permise pour sa réalisation : la force est maximale au point de l'échelle temporelle qui correspond au moment où il reste juste assez de temps avant la date limite pour réaliser la tâche [...]». Shapero qui s'inspire des travaux de Ajzen et Fishbein (1980), soutient que le concept d'intention est le meilleur prédicteur du comportement planifié. C'est pour cette raison qu'il le place au centre de son modèle du processus entrepreneurial. L'événement précipitatif apparaît comme une information jugée déterminante par le dirigeant : il peut jouer un rôle de catalyseur dans le processus de décision d'adoption d'une démarche qualité.

\section{L'articulation entre vision stratégique et intention stratégique}

\subsection{Les étapes du processus d'adoption d'une démarche qualité}

Le processus d'adoption d'une démarche de certification s'intègre normalement dans une perspective plus large. En effet, le fait qu'un dirigeant décide de faire certifier l'entreprise qu'il dirige doit normalement servir un but plus général. C'est pour cette raison que les notions de vision et d'intention stratégiques sont en toile de fond dans la figure 2 .

Le schéma de la page suivante s'efforce d'intégrer le processus d'adoption d'une démarche de certification dans une démarche stratégique globale constituée par l'articulation des notions de vision et d'intention stratégiques. Le dirigeant peut avoir une représentation du processus de certification d'entreprise plus ou moins claire. Sa connaissance est liée à ses expériences passées, à son réseau relationnel et à son écoute de l'environnement. Cette information est plus ou moins disponible selon les secteurs. Dans des secteurs tels que l'automobile et l'armement, les informations concernant l'assurance qualité sont largement répandues. Dans d'autres secteurs, la mise sous assurance qualité reste un phénomène assez récent et limité à des entreprises de grande taille. Dans ce dernier cas, la représentation du dirigeant relative à la mise sous assurance qualité risque d'être floue. Or, lorsque la représentation n'est pas claire, le dirigeant peut avoir du mal à développer ses intentions. 
Figure 2

Cadre conceptuel du processus d'adoption d'une démarche de certification d'entreprise par un dirigeant-propriétaire de petite entreprise

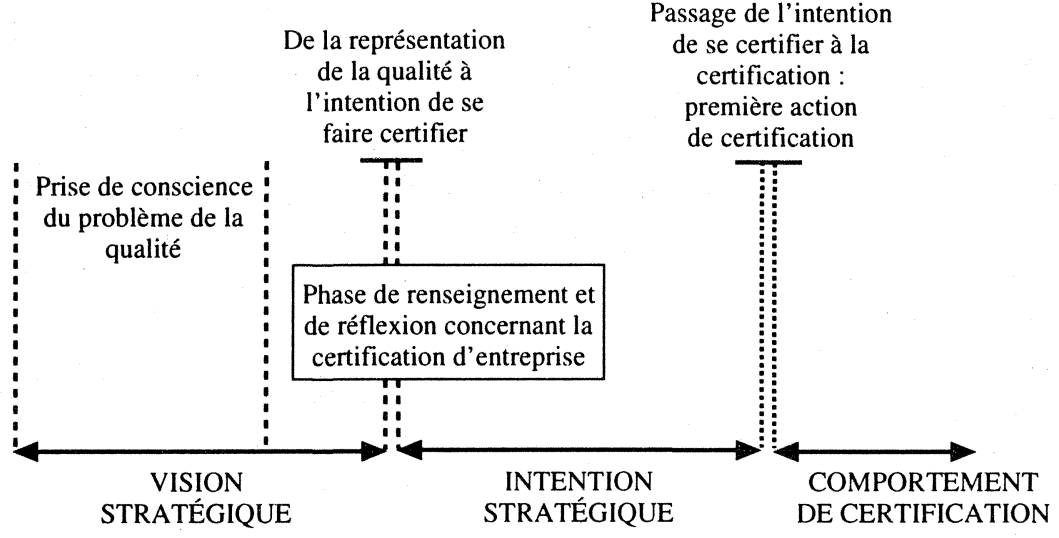

Donc, la probabilité de survenance du comportement risque d'être faible si l'on s'en tient à la théorie du comportement planifié proposée par Ajzen et Fishbein (1980).

La représentation de la qualité pour le dirigeant peut ne constituer qu'une composante de sa vision stratégique. La volonté d'être certifiée peut s'inscrire dans une stratégie plus globale. La mise sous assurance qualité peut apparaître, par exemple, comme un moyen de tisser des relations de partenariat avec certains de ses clients (Baudry, 1995). Pour favoriser le partenariat, l'assurance qualité n'est pas la seule réponse possible. Les entreprises peuvent privilégier la certification produit telle que la norme NF, les labels et autres certificats de conformité. Elles peuvent également mener de front ces différentes démarches.

La définition que nous avons proposée de la vision stratégique ne se limite pas à la seule représentation du futur de l'organisation et de son environnement, mais intègre également la dimension du présent. Le passage de la vision stratégique à l'intention stratégique traduit l'état de tension qui peut résulter du décalage entre la représentation du présent et celle du futur. Cet état de tension a été évoqué par Bird (1988) dans le cadre du processus entrepreneurial. On peut penser que plus cet état de tension est fort et plus l'intention sera forte ; cependant, si cette tension devient trop forte, c'est-à-dire si la représentation de l'état futur paraît inaccessible, alors l'intention deviendra faible et pourra conduire à l'inhibition (Senge, 1991). On peut représenter cette relation sous la forme d'une courbe en $U$ renversé (figure 3). 
FIGURE 3

\section{Relation entre tension et intention}

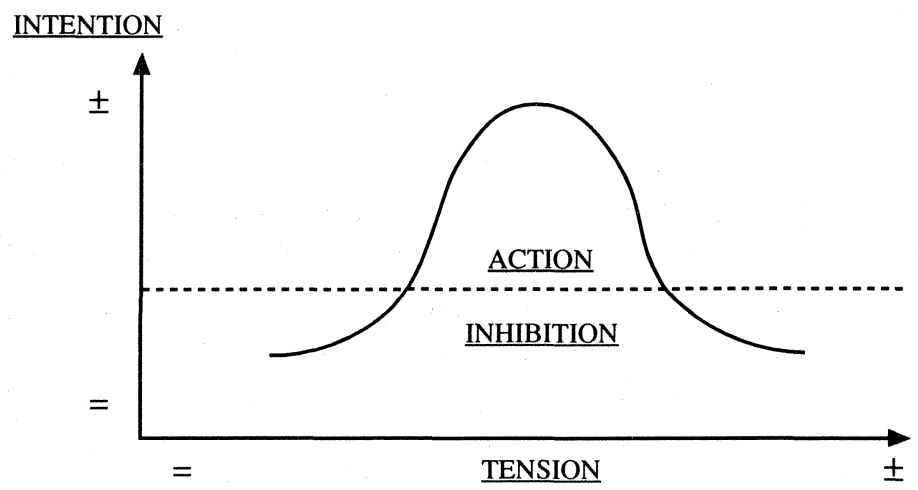

Comment expliquer le passage de la vision stratégique et l'intention stratégique? Ce passage suppose que le dirigeant ait acquis certaines informations relatives à la démarche et à sa mise en œuvre. Deux attitudes vont jouer un rôle de médiateurs entre la vision et l'intention. Il s'agit de la faisabilité perçue et de la désirabilité perçue. La propension à agir joue davantage un rôle de variable modératrice comme l'affirme Kruger (1993), «si la propension à agir est faible, les attitudes seront sans doute moins prédictives de l'intention et de l'action». L'existence d'un événement précipitatif est susceptible d'accélérer la transition de l'intention à l'action. L'événement précipitatif est le plus souvent d'origine externe. Il peut s'agir d'une contrainte, voire d'une circonstance opportune qui impose ou qui encourage l'action. Nous allons essayer de comprendre le processus de décision relatif à l'adoption d'un modèle d'assurance de la qualité en analysant ces quatre concepts.

\subsection{Analyse des quatre variables clés}

La relation complexe qui existe entre vision stratégique, intention stratégique et comportement n'est pas linéaire. En effet, comme l'écrit Avenier (1997), « l'élaboration/mise en acte d'une stratégie repose sur la mise en œuvre tâtonnante d'actions délibérées au sein de situations émergentes ». Filion (1991) décrit la vision stratégique comme le fruit de visions qui émergent. Ainsi, le comportement de mise sous assurance qualité peut exercer une influence sur la vision stratégique et sur l'intention stratégique à travers les difficultés éprouvées, mais également sur les occasions qui se sont présentées à travers cette démarche. Cependant, nous 
souhaitons, dans le cadre de cette contribution, privilégier le processus de décision et non pas la mise en œuvre de l'assurance qualité. Les quatre variables clés sont des concepts qui vont nous permettre d'éclairer le passage entre vision, intention et comportement.

\subsubsection{La faisabilité perçue}

La faisabilité perçue du projet de mise sous assurance qualité repose à la fois sur la perception par le dirigeant de ses capacités à mettre en œuvre cette démarche ainsi que sur sa perception des capacités de son organisation à adopter cette approche.

Le dirigeant peut s'interroger sur la capacité de son entreprise à évoluer. En effet, la mise sous assurance qualité peut constituer une révolution organisationnelle dans la mesure où l'entreprise doit passer d'une culture orale à une culture écrite, doit définir de façon précise les responsabilités de chacun notamment en matière d'obtention de la qualité, doit mettre en place un système d'information interne, doit formaliser sa stratégie qualité et la traduire sous forme de plan. La culture organisationnelle peut constituer un obstacle à la conception d'un système d'assurance de la qualité. Giordano (1997) évoque la difficulté d'actualiser la vision et l'importance du système de pouvoir : « le second ingrédient majeur pour l'action stratégique est bien évidemment le pouvoir. Les modalités d'exercice de celui-ci, le choix des acteurs-clés constituent des ingrédients essentiels pour le pilotage de l'action stratégique et pour la légitimation de celle-ci».

Le rôle du dirigeant de petite entreprise est très important dans la mise en place d'un modèle d'assurance qualité. Par conséquent, la faisabilité perçue dépend dans une large mesure de ses capacités perçues à faire évoluer l'organisation et sa culture. Schein (1990) insiste sur le rôle du dirigeant qui est capable de modifier les formes de représentation des membres de l'organisation grâce à la formation et à la socialisation, particulièrement, dans les entreprises de petite taille. Selon cet auteur, l'élaboration de la culture peut être analysée selon deux mécanismes : à travers les incidents critiques et à travers l'identification au leader: " a second mechanism of culture creation is the modeling by leader figures that permits group members to identify with them and internalize their values and assumptions ». Ce deuxième mécanisme est très prégnant en petite entreprise comme le souligne Mintzberg (1989) dans sa présentation des organisations de type entrepreneurial. Le leader visionnaire est décrit par Mintzberg et Waters (1985) comme « un individu ayant un contrôle personnel de l'organisation et capable d'imposer sa vision ». Il apparaît donc que le succès de la mise sous assurance qualité dépend largement de l'engagement du dirigeant. Pour que le dirigeant se lance dans cette démarche, il doit avoir la volonté de faire évoluer la culture organisationnelle et le fonctionnement organisationnel, mais aussi avoir le sentiment qu'il en est capable (Bird et Jelinek, 1988). Toutefois, cette évolution de l'entreprise risque de poser certaines 
difficultés. On peut identifier trois formes de risque : organisationnel, financier et relationnel. Ces risques sont plus ou moins perçus par le dirigeant. Certains biais cognitifs ont pour effet d'amplifier ces risques, tandis que d'autres biais conduisent à une sous-estimation, voire à une ignorance de ces mêmes risques (Schwenk, 1984).

\subsubsection{La désirabilité perçue}

La désirabilité perçue fait référence à l'attrait du projet pour le dirigeant. Cet attrait peut être analysé soit en référence à l'organisation, soit en référence aux relations avec l'environnement.

La mise sous assurance qualité peut constituer pour l'entreprise un projet mobilisateur. En effet, tous les membres de l'organisation sont concernés et peuvent apporter leur contribution. En outre, le fait d'obtenir un certificat d'assurance qualité peut être perçu par les salariés et le dirigeant comme une consécration, comme la reconnaissance des efforts réalisés pour répondre aux exigences des clients. Le dirigeant peut donc se représenter la certification d'entreprise comme un moyen pour redynamiser l'organisation.

Le dirigeant peut également désirer faire évoluer son entreprise vers un mode de fonctionnement davantage managérial. En effet, l'assurance qualité encourage la mise en place de système de planification et de contrôle, le recours à la standardisation et à la formalisation. Cette stratégie d'adoption d'un modèle d'assurance de la qualité peut s'observer pour des entreprises qui souhaitent consolider leur croissance. Cependant, le dirigeant peut désirer limiter la formalisation, la spécialisation et la standardisation au seul système qualité et donc conserver un mode de fonctionnement entrepreneurial.

La désirabilité perçue peut s'apprécier par rapport aux relations avec l'environnement. Le fait d'appartenir à une organisation professionnelle peut encourager le dirigeant à se lancer dans cette démarche. Par exemple, dans la région LanguedocRoussillon (sud de la France), l'ARIA (Association régionale des industries agroalimentaires) a encouragé ses membres à entreprendre des démarches d'assurance qualité. Les entreprises qui ont obtenu un certificat ISO 9002 ont bénéficié du soutien de cette organisation professionnelle à travers des formations, la constitution de réseaux entre entreprises non concurrentes permettant de réaliser des échanges dans le domaine de la qualité. Parmi la vingtaine d'entreprises certifiées dans cette région, près du tiers sont des petites entreprises indépendantes. Ce résultat montre que ces démarches peuvent être mises en œuvre dans de petites entreprises, mais qu'elles restent encore marginales. On peut donc considérer que le fait de s'être lancé avec succès dans cette démarche s'inscrit dans une stratégie entrepreneuriale. En effet, dans ce secteur d'activité, les entreprises ne se voient pas imposer des modèles d'assurance qualité. Ces entreprises anticipent une évolution des relations interentreprises et souhaitent être les premières à pouvoir répondre aux nouvelles 
exigences pressenties en matière de gestion de la qualité. Pour une petite entreprise agroalimentaire, disposer d'un certificat ISO 9002 constitue encore un avantage concurrentiel dans la mesure où il s'agit d'une démarche lourde et longue à mettre en œuvre. S'agit-il d'un avantage concurrentiel durable ? Selon J. Barney (1991),

Dans certaines circonstances, la première entreprise à mettre en œuvre une stratégie dans une industrie peut obtenir un avantage concurrentiel durable sur les autres entreprises. Cette entreprise peut obtenir l'accès à des canaux de distribution, développer un good-will, se constituer une bonne réputation bien avant que les autres entreprises se lancent dans cette stratégie. Ainsi, le premier (first moving firm) peut accéder à un avantage concurrentiel durable.

\subsubsection{La propension à agir}

Selon Krueger (1993), la propension à agir traduit la capacité du décideur à exercer un contrôle sur ses décisions. Cette notion est assez proche du «locus of control» qui peut être traduit comme le champ de contrôle ou contrôle du destin. Ce concept fait référence à la capacité de l'individu à exercer une influence sur la conduite de sa vie (ou de sa destinée). Ainsi, les actions entreprises par un individu ne sont pas le fait de la chance ou de facteurs externes lorsque celui-ci manifeste un champ de contrôle interne (ou externe). Ce concept développé par Rotter (1966) a donné lieu à de nombreux travaux dans le domaine de l'entrepreneuriat. Les dirigeants caractérisés par un champ de contrôle interne seraient plus à même d'adopter des stratégies entrepreneuriales (McGraw et Robichaud, 1995).

La propension à agir apparaît comme une variable modératrice qui peut conditionner le succès d'une démarche de mise sous assurance qualité. En effet, l'absence de propension à agir peut conduire à l'inhibition comme le suggère Krueger (1993). L'engagement de la direction est souvent évoqué dans la littérature sur la gestion de la qualité comme un facteur de succès. Un chapitre de la norme ISO 9001/2/3 est consacré à la responsabilité de la direction. Dans une enquête réalisée par l'AFAQ, il apparaît que la principale cause de non-conformité lors des audits qu'elle réalise est liée au non-respect de ce chapitre. D'après ce chapitre, le dirigeant doit avoir une vision claire du processus de mise sous assurance qualité et il doit être capable de la traduire sous forme de plans. Il doit donc être en mesure de faire partager sa vision à l'ensemble des membres de l'organisation. Cependant, le dirigeant peut très bien avoir une forte propension à agir tout en ne souhaitant pas jouer un rôle moteur dans le processus de certification, en recrutant, par exemple, un responsable de la qualité.

\subsubsection{L'événement précipitatif}

Le processus de décision de mise sous assurance qualité peut être influencé par un événement précipitatif. L'importance de l'événement précipitatif réduit la dimension entrepreneuriale de cette décision lorsqu'il s'agit d'une contrainte. 
L'évolution de la réglementation peut constituer un événement précipitatif. Par exemple dans l'agroalimentaire, les entreprises européennes sont encouragées à se lancer dans des démarches d'assurance qualité. Ainsi, la directive 93/43 CEE rend pénalement et civilement responsables les producteurs, transformateurs et distributeurs en matière de sécurité alimentaire. Pour exercer cette responsabilité, les fabricants doivent utiliser des outils particuliers de maitrise de la sécurité alimentaire. Cette directive propose notamment de recourir à l'assurance qualité sous sa forme de certification d'entreprise. Notons, cependant, que cette démarche est présentée comme une démarche volontaire.

Dans certaines industries, le maintien de la relation avec les clients peut être conditionné par la détention d'un certificat d'assurance qualité, c'est le cas de l'industrie automobile. Les dirigeants n'ont pas le choix, s'ils souhaitent conserver leurs relations avec leurs principaux clients : ils doivent adopter des modèles d'assurance de la qualité tels que le modèle EAQF (Évaluation d'aptitude qualité des fournisseurs) lancé en 1987 par les constructeurs Renault et PSA-PeugeotCitroën qui est complémentaire au modèle ISO 9001. En outre, les équipementiers ont une faible autonomie de décision. Très souvent, les fournisseurs qui ne sont pas en mesure de répondre à ces exigences sont exclus comme le souligne Gava (1996). Ainsi, en 10 ans, le nombre de fournisseurs de PSA-Peugeot-Citroën est passé de 2200 à 800 , en Europe. Cette tendance à réduire le nombre de fournisseurs encourage les dirigeants de petite entreprise à se lancer dans des démarches d'assurance qualité. Signalons que le fait d'être certifié ne constitue qu' une condition nécessaire, mais non suffisante à l'élaboration de relations durables.

À l'aide de ces quatre concepts, nous avons essayé d'éclairer les relations qui existent entre vision stratégique, intention stratégique et comportement. Toutefois, la relation entre ces concepts ainsi que l'importance de ces concepts dépendent de la nature du processus de décision.

\section{Typologie des processus d'adoption d'une démarche qualité}

L'articulation entre vision stratégique et intention stratégique peut dépendre de la stratégie d'adoption d'un modèle d'assurance de la qualité. La démarche de certification d'entreprise constitue un investissement immatériel. Guillon (1993) montre qu'il est possible de distinguer deux types de processus de prise de décision d'adoption d'un investissement immatériel selon l'attitude proactive ou réactive du dirigeant de petite entreprise. Ces deux processus doivent être perçus comme les extrémités d'un continuum. Nous allons nous appuyer sur le cadre conceptuel que nous avons proposé pour analyser ces deux principales stratégies. Nous illustrerons cette réflexion à partir de deux mini-études de cas effectuées à partir d'entretiens réalisés avec les dirigeants de deux entreprises agroalimentaires. 


\subsection{Stratégie proactive}

Bateman et Grant (1993) ont proposé une définition de l'attitude proactive qui est la suivante :

The prototypic proactive personality, as we conceive it, is one who is relatively unconstrained by situational forces, and who effects environmental change. [...] Proactive people scan for opportunities, show initiative, take action, and persevere until they reach closure by bringing about change. They are pathfinders (Leavitt, 1988) who change their organization's mission or find and solve problem.

Le processus proactif est caractéristique des stratégies entrepreneuriales. Selon Mintzberg (1973), les stratégies entrepreneuriales peuvent être définies par la recherche active de nouvelles occasions d'affaires. Cette recherche peut se traduire par le lancement de nouveaux produits, l'adoption de nouvelles technologies ou de nouveaux modes de gestion.

La figure 4 illustre le processus proactif. Elle met en évidence l'enchaînement entre vision stratégique, intention stratégique et mise sous assurance qualité.

FIGURE 4

Processus de décision de type proactif

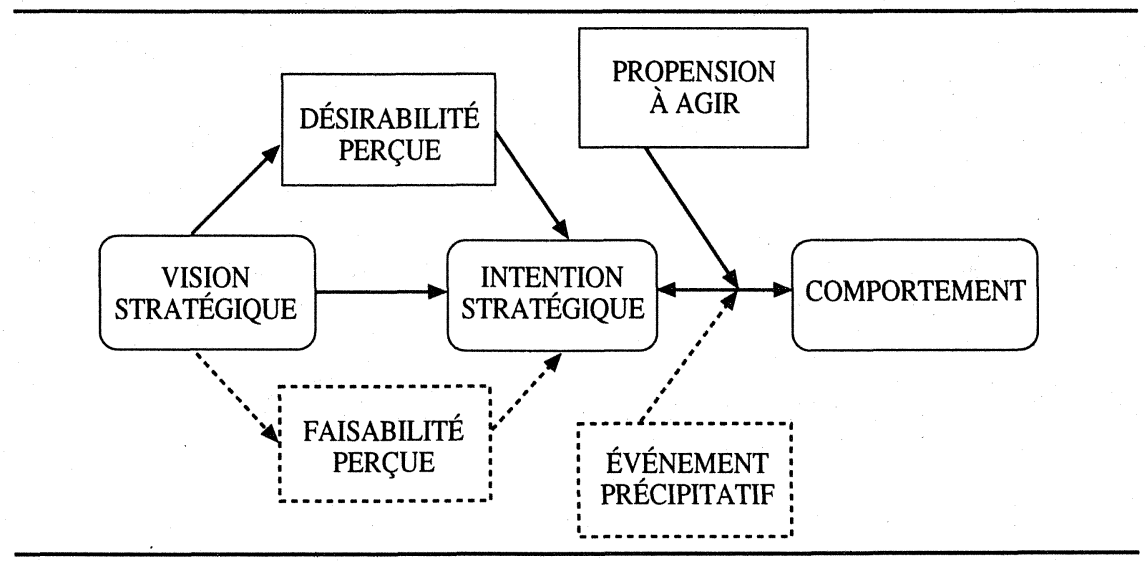

La vision stratégique du dirigeant va jouer un rôle très important. Si le dirigeant considère que la certification d'entreprise peut être un moyen de se constituer un avantage concurrentiel, la désirabilité sera forte. La faisabilité perçue ne va pas jouer un rôle déterminant dans la mesure où il va avoir tendance à considérer qu'il est en mesure de faire évoluer les choses. Dans certains cas, le dirigeant peut même avoir tendance à éluder les risques organisationnels ou financiers suscités par la mise sous assurance qualité. Palich et Bagby (1995) ont vérifié l'existence de ces biais cognitifs chez les entrepreneurs : «In accord with cognitive theory, we averred 
that entrepreneurs may not actually prefer to take risks; rather, due to schema accessibility, they simply tend to associate business situations with cognitive categories that suggest more favorable attributes. » Schwenk (1984) évoque ce biais cognitif dans le processus de décision sous le terme d'illusion de contrôle.

On risque d'observer une forte propension à agir notamment lorsque peu d'entreprises se sont lancées dans la démarche. Dans les industries agroalimentaires, les entreprises qui se sont lancées dans une démarche d'assurance qualité ne se sont pas vu imposer la mise sous assurance qualité. Elles ont bénéficié de la part des organisations professionnelles d'informations sur la démarche et sur les possibilités de financements, comme la plupart des autres entreprises. Les dirigeants de ces entreprises ont envisagé un durcissement des relations interentreprises dans ce secteur ou se sont lancés dans cette démarche pour promouvoir un partenariat avec leurs clients.

Les événements précipitatifs ne vont pas jouer un rôle important dans le passage de l'intention au comportement. L'entrepreneur n'attend pas un message explicite de l'environnement pour déclencher le processus de certification. Bien au contraire, il l'anticipe. Il est à l'écoute de son environnement ; il n'hésite pas à jouer un rôle actif dans les organisations professionnelles pour disposer d'informations et pour se créer un réseau relationnel. La mini-étude de cas ci-dessous illustre ce processus proactif.

\subsection{Stratégie réactive}

Bateman et Grant (1993) préfèrent parler d'attitude passive qu'ils définissent comme : "Other people, who would not be so classified, are relatively passive they react to, adapt to, and are shaped by their environments. [...] They show little initiative, and rely on others to be forces for change. "

Le processus de prise de décision est caractéristique de la stratégie adaptative. Lorsque le dirigeant a plutôt une attitude réactive (figure 5), la vision stratégique et l'intention stratégique ne semblent pas jouer un rôle déterminant. L'événement précipitatif est au cœur du processus de prise de décision et peut prendre la forme d'un durcissement des conditions d'achat imposées par certains clients, notamment sur le plan de la qualité. Le dirigeant se doit alors d'enclencher cette démarche s'il souhaite assurer la continuité de son entreprise. Sa marge de liberté est beaucoup plus faible.

La désirabilité perçue peut être influencée par l'événement précipitatif. Par exemple, le fait qu'un concurrent soit certifié peut inciter le dirigeant à se lancer dans une démarche d'assurance de la qualité. La mise aux normes peut constituer un défi pour le dirigeant.

La faisabilité perçue ne va pas jouer un rôle important dans le processus de décision. En effet, il ne s'agit pas, pour le dirigeant, de savoir si cette démarche est adaptée à l'entreprise, mais de savoir comment répondre à ces nouvelles exigences. 


\section{Mini-étude de cas 1}

Monsieur A. a repris l'entreprise familiale après avoir travaillé dans un service marketing d'une grande entreprise électronique. Cette entreprise de la région de Nîmes a connu de graves inondations en 1989. Le dirigeant a décidé de construire une nouvelle unité qui répond aux normes $\mathrm{CEE}$. Cette entreprise a donc été l'une des premières à bénéficier de l'agrément CEE en 1991. Cette mise aux normes très rapide a eu des effets très positifs en termes de réputation tant auprès des circuits de distribution que de certains grands concurrents pour lesquels elle réalise de la sous-traitance de capacité. Cet avantage concurrentiel n'étant pas durable, M. A. a envisagé de se lancer dans une démarche d'assurance qualité. Selon lui, la mise aux normes ISO 9002 devait lui permettre d'atteindre quatre objectifs : la pérennité, la diminution des coûts de nonqualité, l'élaboration d'un projet d'entreprise et la différenciation. Cette volonté d'adopter la norme ISO 9002 s'inscrivait dans une intention stratégique plus large marquée par le désir de tisser des relations durables avec la grande distribution. La faisabilité perçue du projet ne semble pas avoir joué un rôle important dans son processus de prise de décision. La représentation qu'il avait de cette démarche s'appuyait sur des informations relativement imprécises. En outre, la formulation très vague des normes ISO 9000 ne permet pas de se représenter précisément les implications organisationnelles d'une telle démarche. En 1992, M. A. a lancé ce projet. Il a fallu deux ans pour sensibiliser le personnel, expliquer la démarche. Le manque de moyen ne permettait pas d'atteindre l'objectif. M. A. a alors décidé d'intensifier ses relations avec l'ARIA pour bénéficier de leur soutien. Il a recruté un responsable qualité pour se faire aider dans cette tâche. Dix-huit mois plus tard, cette entreprise de 26 salariés obtenait le certificat ISO 9002. Selon M. A., les deux premières années ont été indispensables dans la mesure où il a fallu faire évoluer les représentations de l'ensemble des membres de l'organisation. Ce bouleversement de la culture d'entreprise explique pourquoi la deuxième phase n'a nécessité finalement que 18 mois.

FIGURE 5

Processus de décision de type réactif

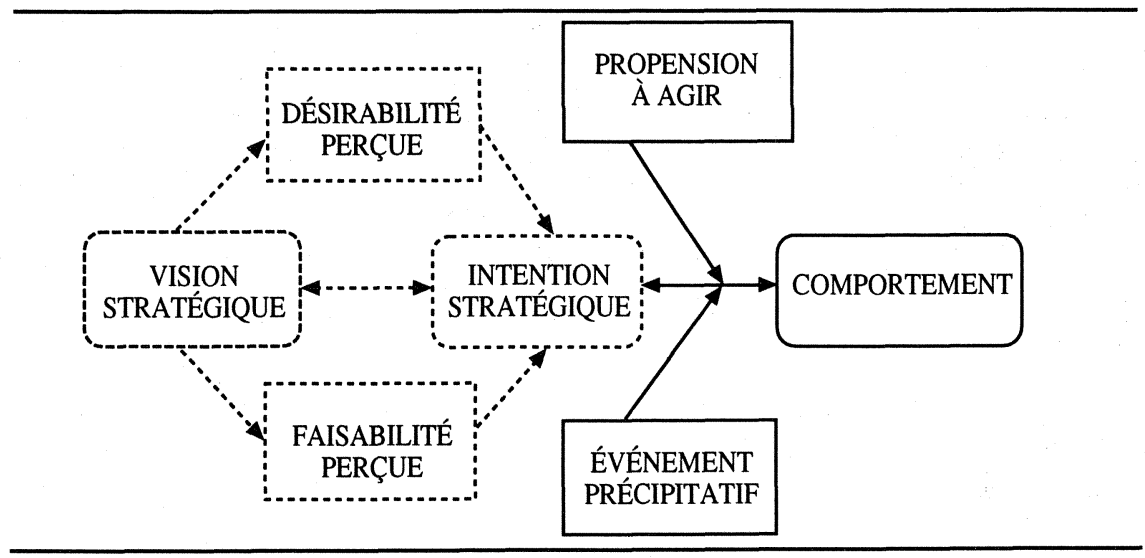

Revue internationale P.M.E., vol. 11, nº 1, 1998 
La propension à agir est faible en l'absence d'événement précipitatif et devient forte en sa présence. Dans le schéma ci-dessus, nous avons préféré parler de propension à réagir. Ainsi, le dirigeant peut avoir la volonté de se lancer dans cette démarche et s'impliquer pour faire aussi bien qu'un concurrent ou pour répondre à de nouvelles exigences de ses clients.

\section{Mini-étude de cas 2}

Monsieur Z. se décrit comme un fils d'agriculteur qui croit aux valeurs de la terre, à la bonne cuisine. Il a repris, il y a quelques années, l'entreprise familiale qui fabrique des plats cuisinés et des produits typiques de la région nîmoise. La mise aux normes CEE a été vécue comme un passage obligé qui a nécessité un lourd investissement d'environ $6 \mathrm{MF}$. Les charges financières s'élèvent à $150000 \mathrm{~F}$ par mois. M. Z est contraint de privilégier la croissance de son affaire pour amortir ses investissements et pour atteindre une certaine taille pour pouvoir pérenniser ses relations avec la grande distribution. Il souhaite doubler ou tripler son chiffre d' affaires. À l'origine, ils étaient trois ; maintenant, ils sont huit. Ils regrettent le temps où les relations étaient plus familiales et moins revendicatives. Il a embauché une personne qui s'occupe davantage de la production, car il ne souhaite plus participer aux tâches de fabrication. Il préfère se consacrer aux activités commerciales. Il n'a pas hésité à participer au SIAL et à investir $50000 \mathrm{~F}$ dans cette manifestation. Il a obtenu une cinquantaine de contacts dont une dizaine semblent prometteurs notamment à l'exportation. Pour intensifier ses relations avec la grande distribution, il a recruté deux vendeurs multicartes dont l'un est un ancien responsable des achats chez Carrefour. Il ne se sent pas à la hauteur pour aller négocier avec la grande distribution et souhaite bénéficier de l'expérience et du réseau de relations de ses vendeurs. Cette phase d'apprentissage lui semble indispensable pour connaître tous les secrets du référencement et pour se créer à son tour un réseau. Son entreprise réalise $20 \%$ de son chiffre d'affaires avec la grande distribution et souhaite réaliser $60 \%$ dans les années à venir. La mise aux normes CEE oblige une plus grande rigueur en matière de gestion de la qualité essentiellement dans le domaine de l'hygiène. M. Z. a dû mettre en place la méthode HACCP en faisant appel à un cabinet de consultant pour répondre aux exigences de la directive 93/43 CEE. Il envisage cette année de se lancer dans une démarche d'assurance de la qualité. L'un de ses principaux concurrents a obtenu, il y a quelques mois, son certificat ISO 9002. Il a le sentiment que cette norme devient un passage obligé notamment pour être présent en grande distribution. Ses clients n'ont pas manifesté cette exigence, toutefois, la référence aux normes ISO 9000 apparaît de façon récurrente dans les médias, dans les discussions entre professionnels. L'événement précipitatif a été l'adoption de la norme ISO 9002 par l'un de ses concurrents. Pour lui, il ne s'agit pas de se différencier, mais d'éviter de se marginaliser. Il n'apprécie pas cette évolution de la réglementation dictée, selon lui, par certaines grandes chaînes d'alimentation rapide. Il subit l'industrialisation de son activité qui se traduit par une plus grande automatisation, un plus grand recours aux procédures. 


\section{Conclusion}

L'adoption d'un modèle d'assurance de la qualité constitue un enjeu pour de nombreuses PME. Les dirigeants s'interrogent sur l'opportunité de retenir une telle démarche en raison notamment de sa lourdeur et de son coût. Nous avons essayé dans le cadre de cette contribution de saisir ce processus de décision en retenant une approche cognitive. Le modèle de Shapero permet de mieux comprendre les décisions des dirigeants qui peuvent revêtir une dimension entrepreneuriale. Le cadre d'analyse proposé peut donc être transposé à de nombreuses décisions stratégiques. Nous avons particulièrement insisté sur les notions de vision et d'intention stratégiques. La certification d'entreprise peut correspondre à une dimension importante de la vision stratégique, mais également constituer un moyen dans la réalisation de l'intention stratégique.

Notre contribution, qui s'est attachée à être une réflexion conceptuelle, débouche sur une grille d'analyse qui peut être considérée comme un modèle ; nous envisageons de le tester auprès de dirigeants-propriétaires de petite entreprise. De nombreux auteurs (Shapero, 1982 ; Carrière, 1990 ; Krueger, 1993 ; NkongoloBakenda, D'Amboise et Garnier, 1994 ; Larwood et al., 1995) ont proposé des variables permettant de mesurer les concepts qui figurent dans notre modèle, et que nous souhaitons retenir dans le cadre de notre enquête.

Ce cadre d'analyse peut se révéler particulièrement intéressant dans la construction d'études de cas. Le recours à la cartographie cognitive peut alors permettre d'élucider les différents éléments proposés dans la grille d'analyse et notamment d'apprécier la cohérence stratégique du projet formulé par le dirigeant. Les travaux de Cossette $(1993,1994)$ consacrés à l'analyse de la vision stratégique s'inscrivent dans ce courant de recherche. L'analyse que nous avons conduite dans cet article nous a ainsi permis de mettre en évidence la diversité des stratégies d'adoption d'une démarche qualité en PME.

\section{Bibliographie}

AHIRE, S.L. et D.Y. GolHAR (1996), «Quality management in large vs small firms », Journal of Small Business Management, avril.

AJZEN, I. (1991), « The theory of planned behavior », Organizational Behavior and Human Decision Process, vol. 50.

AJZEN, I. et M. FISHBEIN (1980), «Attitudes traits, and actions, dispositional prediction of behavior in social psychology », Advances in Experimental Social Psychology, vol. 20.

AVENIER, M. J. (1997), «Une conception de l'action stratégique en milieu complexe : la stratégie tâtonnante», dans M.J. Avenier, La stratégie «chemin faisant », Paris, Economica. 
BARNEY, J. (1991), «Firm resource and sustained competitive advantage», Journal of Management, vol. 17, $\mathrm{n}^{\mathrm{o}} 1$.

BATEMAN T. S. et J. M. GRANT (1993), «The proactive component of organizational behavior : a measure and correlates", Journal of Organizational Behavior, vol. 14.

BAUDRY, B. (1995), L'économie des relations interentreprises, Paris, Éd. La Découverte.

BENNIS, W. (1982), « Leadership transforms vision into action », Industry Week, vol. 213.

BIRD, B. et M. JELINECK (1988), «The operation of entrepreneurial intentions», Entrepreneurship Theory and Practice, vol. $13, \mathrm{n}^{\circ} 2$, hiver.

BIRD, B. (1988), «Implementing entrepreneurial ideas : the case for intentions », Academy of Management Review, vol. 13.

CARrIÈre, J.B. (1990), «La vision stratégique en contexte de PME : cadre théorique et étude empirique», Revue internationale PME, vol. 3, $\mathrm{n}^{\text {os }}$ 3-4.

COLLINS, J.C. et J.I. PORRAS (1991), «Organizational vision and visionary organizations », California Management Review, vol. 34, $\mathrm{n}^{\circ}$ 1, automne.

CossetTe, P. et M. Audet (1994), «Qu'est-ce qu'une carte cognitive», dans P. Caussette (éd.), Cartes Cognitives et Organisations, Paris, Édition Eska.

COSSETTE, P. (1993), «La vision stratégique du propriétaire-dirigeant de PME : Une étude de cartographie cognitive ", dans Compétivité des PME et marchés sans frontières, Congrès international francophone de la PME, Carthage, octobre.

COSSETTE, P. (1994), «Développement d'une méthode systématique d'aide à la mise de la vision stratégique chez le propriétaire dirigeant de PME : une étude exploratoire », Actes de la $39^{\circ}$ conférence de l'ICSB, Strasbourg.

COULSON-THOMAS, C. (1992), «Strategic vision or strategic con ? Rhetoric or reality », Long Range Planning, vol. $25, \mathrm{n}^{\mathrm{0}} 1$.

D'AMBoise, G. et S. BouchaRD (1990), « De l'utilisation du concept de vision dans l'étude des organisations », Document de travail 90-02, Université Laval.

D’AMBoISE, G. et Y. GASSE (1989), «Vision stratégique et activités de planification : pour un modèle différent de recherche en $\mathrm{PME}$ », Communication au XXXIV ${ }^{\mathrm{e}}$ congrès du Conseil international de la petite entreprise, Québec, juin.

DÖRNER, M. (1986), «Intention memory and intention regulation», dans F. Klix et H. Hagendorf (éd.), Human Memory and Cognitive Capabilities, Mechanisms and Performance, Elsevier, Science Publishers.

ESPOSITO, E. et M. RAFFA (1996), «L'évolution de la gestion de la qualité totale dans les petites entreprises sous-traitantes du secteur de l'aéronautique », Revue internationale PME, vol. $9, \mathrm{n}^{\circ} 3$.

FILION, L.J. (1989), «Le développement d'une vision : un outil stratégique à maîtriser », Revue internationale PME, vol. $2, \mathrm{n}^{\circ} 3$.

GAVA, M.J. (1996), «Les équipementiers dans la danse de la certification », Enjeux, n 169.

GHOBADIAN, A. et D.N. GALlEAR (1996), « Total quality management in SMEs », Omega, International Journal of Management Science, vol. 24, $\mathrm{n}^{0} 1$. 
GIORDANO, Y. (1997), «L'action stratégique en milieu complexe : quelle communication?», dans M.J. Avenier, La stratégie «chemin faisant », Paris, Economica.

GRATTON, L. (1994), «Implementing strategic intent : human resources processes as a force for change », Business Strategic Review, vol. 5, $\mathrm{n}^{\circ} 1$.

GREFFEUILle, J. (1994a), «Comment aider à la formulation d'une vision stratégique? Fondements théoriques et méthodes », IAE Aix-Marseille, Document de travail $\mathrm{n}^{\circ} 421$.

GrefFEUILLE, J. (1994b), «Comment aider à la formulation d'une vision stratégique ? Illustration de la démarche », IAE Aix-Marseille, Document de travail n ${ }^{\circ} 422$.

GREFFEUILLE, J. (1994c), « Comment opérationnaliser la formulation d'une vision stratégique?», IAE Aix-Marseille, Document de travail no 423.

GuILLON, A. (1993), Étude de la relation entre le changement organisationnel et l'investissement intellectuel dans les PME, Thèse de doctorat en science de gestion, Université de Montpellier I.

Guillon, A. et M. WeILL (1997), «Les démarches qualité en PME : méthodologies et résultats d'enquête », Actes de la Ve conférence de l'Association internationale de management stratégique, Montréal.

Gunasekaran, A., P. OKKo, T. Martikainen et P. Yli-Olli (1996), «Improving productivity and quality in small and medium enterprises : cases and analysis », International Small Business Journal, vol. 15, $\mathrm{n}^{\circ} 1$.

HAmel, G. et C.K. Prahalad (1989), «Strategic intent», Harvard Business Review, mai-juin.

HAREL GiASSON, F. (1995), «Faut-il vraiment avoir de la vision?», Gestion, vol. 20, no 3.

JULIEN, P.A. (1994), «Pour une définition des PME», dans P.A. Julien (éd.), Les PME: Bilan et perspectives, Paris, Économica.

KRUEGER, N. (1993), «The impact of prior entrepreneurial exposure on perceptions of new venture feasibility and desirability », Entrepreneurship Theory and Practice, vol. 18, $\mathrm{n}^{\circ} 1$, automne.

LAROCHE, H. et J.P. NIOCHE (1994), «L'approche cognitive de la stratégie d'entreprise», Revue française de gestion, $\mathrm{n}^{\circ} 99$.

LARWOOD, L., C.M. FALBE, M.P. KRIGER et P. MIESNING (1995), « Structure and meaning of organizational vision », Academy of Management Journal, vol. 38, n 3.

LEAVITT, H. (1988), Managerial Psychology: Managing Behavior in Organizations, Chicago, Dorsey Press.

MARChESNAY, M. (1991), « La PME : une gestion spécifique », Écomonie Rurale, nº 206.

MCGRAW, E. et Y. ROBICHAUD (1995), «Le concept de contrôle de destin et son influence sur le comportement entrepreneurial », Revue internationale PME, vol. 8, $\mathrm{n}^{\circ} 2$.

MEEKINGS, A., J. DRANSFIELD et J. GodDARD (1994), «Implementing strategic intent: the power of an effective business management process », Business Strategy Review, vol. 5, hiver. 
MESSEGHEM, K. (1997), «L'assurance qualité comme facteur de coopération entre entreprises de grande distribution alimentaire et PME agro-alimentaires », Présentation au colloque international organisé par Nancy II, Renne I, Bologne et Luxembourg, Connivences d'acteurs, contrats, coopération et métamorphose des organisations, Nancy, Luxembourg, mai.

MiNTZBERG, H. et J.A. WATERS (1985), «Of strategies, deliberate and emergent », Strategic Management Journal, vol. 6.

MinTzBerg, H. (1973), «Strategy-making in three modes », California Management Review, vol. XVI, $\mathrm{n}^{\circ} 2$, hiver.

MinTZBERG, H. (1989), Le Management, Paris, Les Éditions d'Organisation.

NGUYEN-XUAN, A., J.F. RICHARD et J.M. HoC (1990), «Le contrôle de l'activité », dans J.F. Richard, C. Bonnet et R. Ghiglione, Traité de psychologie cognitive : Le traitement de l'information symbolique, Paris, Dunod.

Nkongolo-BAKENDA, J.M., G. D' AMBoISE et B. GARNIER (1994), «L'association entre la manifestation de la vision stratégique des propriétaires-dirigeants de PME et la performance de leur entreprise», Revue internationale PME, vol. 7, $\mathrm{n}^{\circ} 1$.

PALICH, L.E. et D.R. BAGBY (1995), «Using cognitive theory to explain entrepreneurial risk-taking : challenging conventional wisdom », Journal of Business Venturing, vol. 10 , p. 425-438.

PARIKH, J. et F. NEUbAUER (1993), «Corporate visioning », International Review of Strategic Management, vol. 4.

RAMANANTSOA, B. (1993), «Les nouvelles approches stratégiques : un défi pour le contrôle de gestion", Problèmes économiques, $\mathrm{n}^{\circ}$ 2, p. 317 , mars.

ROTTER, J.B. (1966), «Generalized expectancies for internal versus external control of reinforcement : a case history of a variable», Psychological Monographs, vol. 80, $\mathrm{n}^{\mathrm{o}} 1$.

SCHEIN, E.H. (1990), «Organizational culture», American Psychologist, vol. 45, nº 2.

SCHOEMAKER, P.J. (1992), «How to link strategic vision to core capabilities», Sloan Management Review, automne.

SCHWENK, C.R. (1984), « Cognitive simplification process in strategic decision-making », Strategic Management Journal, vol. 15.

SEARLE, J.R.(1984), «La structure de l'action», dans Du cerveau au savoir, Hermann, Éditeurs des Sciences et des Arts, coll. «Savoir».

SENGE, P. (1991), La cinquième discipline, Paris, Édition First.

SHAPERO, A. (1982), « Social dimensions of entrepreneurship », dans Kent et al. (éd.), The Encyclopedia of Entrepreneurship, Englewood Cliffs, NJ, Prentice-Hall.

SMITH, C. (1994), «The Merlin factor : leadership and strategic intent », Business Strategy Review, vol. $5, \mathrm{n}^{\mathrm{o}} 1$, printemps.

TREgoe, B., J. ZimMERMAN, R. SMITH et P. TOBIA (1991), La vision stratégique en action, Paris, Les Éditions d'Organisation. 
VARELA, F.J. (1996), «Approche de l'intentionnalité : de l'individu aux groupes sociaux », dans L'organisation apprenante : l'action productrice de sens, tome 1, Université de Provence, Département de science de l'éducation.

WESTLEY, F. et H. MINZBERG (1989), « Visionary leadership and strategic management », Strategic Management Journal, vol. 10.

ZALEZNIK, A. (1977), «Managers and leaders : are they different?», Harvard Business Review, vol. 55, mai-juin. 